Archives de sciences sociales des religions

166 | Avril-Juin 2014

Leszek Kołakowski

\title{
Un sceptique inconséquent. Leszek Kołakowski
}

\section{Paul Zawadzki}

\section{(2) OpenEdition}

Journals

Édition électronique

URL : http://journals.openedition.org/assr/26077

DOI : $10.4000 /$ assr. 26077

ISSN : $1777-5825$

Éditeur

Éditions de l'EHESS

Édition imprimée

Date de publication : 10 juillet 2014

Pagination : 143-152

ISBN : 978-2-7132-2432-4

ISSN : 0335-5985

Référence électronique

Paul Zawadzki, « Un sceptique inconséquent. Leszek Kołakowski », Archives de sciences sociales des religions [En ligne], 166 | Avril-Juin 2014, mis en ligne le 10 juillet 2017, consulté le 21 décembre 2020 URL : http://journals.openedition.org/assr/26077 ; DOI : https://doi.org/10.4000/assr.26077

Ce document a été généré automatiquement le 21 décembre 2020.

(C) Archives de sciences sociales des religions 


\title{
Un sceptique inconséquent. Leszek Kołakowski
}

\author{
Paul Zawadzki
}

\section{RÉFÉRENCE}

Jacques Dewitte, Kołakowski, Le clivage de l'humanité, Paris, Michalon, 2011, 126 p.

Czas ciakawy, Czas Niespokojny. Z Leszkiem Kołakowskim rozmawia Zigniew Mentzel, [Temps intéressant, temps inquiétant. Entretiens avec Leszek Kołakowski par Zbigniew Mentzel], Cracovie, Znak, 2008, 2 vol., 275 et 198 p.

Marcin Król, Czego nas uczy Leszek Kołakowski [Ce que nous apprend Leszek Kołakowski], Varsovie, Czerwone i czarne, 2010, 271 p.

Leszek Kołakowski, Wśród znajomych. 0 różnych ludziach mĄdrych, zacnych, interesujących $i$ o tym jak czasy swoje urabiali [Dans l'entourage des amis. Sur des gens intelligents, vertueux, intéressants et sur la manière qu'ils avaient de se débrouiller avec leur temps], textes choisis et postfacés par Zbigniew Mentzel, Cracovie, Znak, 2006, 204 p.

1 En dépit de l'excellent texte de Jacques Dewitte, petit en volume mais nourri de plus de trente ans de compagnonnage intellectuel ${ }^{1}$, l'œuvre de L. Kołakowski reste sousestimée et mal connue en France. La langue n'en constitue plus l'obstacle majeur. Les traductions en français ou en anglais sont nombreuses. De plus, L. K. écrivait aussi en français, en allemand, avec moins de plaisir en anglais, si bien que la plupart de ses textes importants sont désormais accessibles aux non-polonophones. Ses écrits sur la religion sont commentés depuis longtemps par les Archives en sciences sociales des religions ou par Esprit. Le Kołakowski politique ne nous est pas non plus tout à fait inconnu. Entré au chapitre " révisionnisme » dans les histoires du communisme, figure centrale de l'opposition polonaise jusqu'à la chute du mur de Berlin, L. K. avait noué, dès les années 50 , des liens personnels avec les intellectuels gravitant autour de la revue Arguments (Henri Lefebvre, Edgar Morin, Dionys Mascolo...). Ses textes étaient discutés dans les cercles de la gauche antitotalitaire des années 70 et par les analystes $\mathrm{du}$ communisme, surtout depuis la publication de sa monumentale histoire du 
marxisme ${ }^{2}$. Au tournant des années 1970/80, Commentaire publie sa conférence donnée au Collège de France en 1980 (« Où sont les barbares? Les illusions de l'universalisme culturel »), bientôt suivie de sept ou huit autres articles. Le débat lui fait une place dès son premier numéro avec un texte sur Isaiah Berlin.

2 La sous-estimation ainsi que la réception fragmentaire de l'œuvre de L. K., tiennentelles à la longévité de schèmes de pensée issus du communisme français qui ont longtemps rendu suspect un auteur qui prend Marx au sérieux, mais n'en avance pas moins la thèse des « racines marxistes du stalinisme ${ }^{3}$ "? Le léninisme et le stalinisme ne sont pas davantage des corruptions du marxisme qu'ils n'ont été produits de manière causale par la théorie de Marx. Chacun de nous - écrit Kołakowski - est suffisamment marxiste pour admettre qu' « aucune société n'a jamais été entièrement engendrée par une idéologie et ne peut jamais être expliquée par les idées des hommes qui ont contribué à la faire naître ». Aucun mouvement politique ou religieux ne constitue l'incarnation parfaite d'une essence prétendument contenue dans ses écrits. Pour autant, les idées ne sont pas seulement dictées par les circonstances et privées de toute efficacité, sinon pourquoi en étudierait-on l'histoire? La pensée de Marx ainsi que ses mises en pratique ultérieures s'articulent autour de noyaux métaphysiques stables. Le point de vue adopté par Kołakowski trouve sa comparaison chez Thomas Mann explorant dans Le Docteur Faustus ce qui dans la culture allemande avait rendu possible l'émergence du mouvement hitlérien et de l'idéologie nazie. Son Histoire $d u$ marxisme se donne pour tâche de rendre intelligible cette « étrange histoire d'une idée qui commença avec l'humanisme prométhéen pour finir avec les abominations de la tyrannie stalinienne ». Indépendamment des intentions de Marx, dans quelle mesure le socialisme despotique constitue-t-il l'incarnation de la logique interne de sa doctrine, nouée notamment autour de la théorie de l'aliénation et du concept eschatologique de l'homme unifié4 ? L'idéologie stalinienne appelée à justifier le système stalinien d'organisation sociale fut-elle une interprétation légitime (mais non la seule possible) de la philosophie marxiste de l'histoire? Toute tentative pour mettre en pratique les valeurs fondamentales du socialisme marxien devait-elle engendrer une organisation politique comportant des traits incontestablement analogues au stalinisme ? À ces questions, posées dans "Les racines marxistes du stalinisme», la réponse de Kołakowski est affirmative.

3 Aggravant son cas, l'irréductible auteur de «Comment être socialiste-conservateurlibéral. Credo" (Aneks, 1978, tr. Commentaire, n 4/1978-79) n'est jamais là où on l'attend. Tant par son style, son humour logique que le fond de sa pensée, celui qui se présentait volontiers comme un sceptique inconséquent (non-relativiste) se plait à brouiller les pistes. Pourfendeur des mythes communistes, cet auteur que l'on appellerait « dissident » s'il n'avait refusé ce terme ${ }^{5}$, récuse pourtant les «illusions de la démythologisation ${ }^{6} »$. Loin de se contenter d'une critique du totalitarisme largement accomplie au tournant des années 1960/1970, il en est venu à s'interroger sur les fragilités internes de la modernité et sur «l'auto-empoisonnement des sociétés ouvertes $^{7} »$. Héros intellectuel de la révolte étudiante polonaise de 1968, il n'eut de mots assez durs pour qualifier le kitsch et "l'obscurantisme» du gauchisme estudiantin de Berkeley (Entretiens, vol.2, p.30). Dans Les principaux courants du marxisme, il voyait en Marcuse un hériter des théocrates du Moyen Âge, voire «le prophète de la forme la plus irrationnelle de l'anarchisme semi-romantique ${ }^{8}$ ", critiques certes féroces, mais pas sous-tendues par une normativité « réactionnaire». 
Dernier exemple de chasse-croisé Est/Ouest, à lire Religion. If there is no God... (tr. fr. Philosophie de la religion, Fayard, 1985), on débouche sur l'idée qu'un monde avec Dieu offre plus de sens qu'un monde qui en est privé. L. K. ne propose pourtant pas un retour vers la religion, la comparaison avec Soljenitsyne ne vaut pas. Il pose le vaste problème foi/raison à partir d'une position philosophique soulignant que logiquement, les deux plans ne devraient pas entrer en collision : " prouver sa foi est une contradiction dans les termes » (Philosophie de la religion, p. 273). Sans doute n'aurait-il pas dit avec la même conviction qu'Aron qu'il était " resté un homme des Lumières ", mais sa trajectoire fait cependant songer à celle de l'auteur de L'opium des intellectuels déclarant, dans l'épilogue de ses Mémoires, que l'horreur des religions séculières prétendant au monopole des valeurs ultimes, lui avait rendu quelque sympathie pour les religions transcendantes. Il reste que les rapports au religieux des intellectuels dans la seconde moitié du $\mathrm{xx}^{\mathrm{e}}$ siècle ne sauraient être identiques en France et en Pologne et que de telles perspectives peinent peut-être à trouver leur public dans une société française constituée dans l'affrontement de deux universalismes concurrents - rationalisme des Lumières françaises/universalisme chrétien.

4 Mais l'essentiel, est sans doute ailleurs, à savoir la difficulté de cerner une pensée dont la culture impressionne et la pluralité des domaines déroute. Allant de la politique, à l'éthique, de la philosophie positiviste à celle de la religion, L. K. semble chez lui aussi bien quand il analyse la pensée des croyants sans Église du XvII e siècle que lorsqu'il retrace le sinueux parcours de l'idée socialiste. Délicat aussi de parler de l'unité de l'œuvre: non seulement en raison de son évolution, notamment sur la question du religieux, mais aussi parce que L. K., qui avait été l'ami d'Isaiah Berlin à All Souls College - il leur arrivait de discuter en russe - se pensait davantage comme un "renard » qu'un "hérisson» (Entretiens, vol. 2, p. 49). Le genre essayiste lui convenait à merveille. Le style même, alliant ironie sans cynisme, humour et érudition avec un souci de rigueur logique poussé aux limites du rire, conjugue satire et méditations métaphysiques, histoire des idées et contes religieux.

5 On doit aux ouvrages présentés ici le remarquable effort de restitution de l'ensemble d'une œuvre et d'un chemin intellectuel échappant à l'esprit de système. Compte tenu de l'importance accordée par L.K. à l'étude des conditions de possibilité de toute société/culture (sens, vérité, mythes, croyances, tabous, etc.), on saisit sans peine les raisons qui poussèrent $\mathrm{J}$. Dewitte à chercher la cohérence de sa pensée «non pas à proprement parler dans une métaphysique, une philosophie morale ou une pensée politique, mais dans une anthropologie philosophique et politique ", quand bien même sa conception anthropologique, morale et politique "n'a jamais été énoncée de manière systématique » (J. Dewitte, p. 95). En affinité avec cette perspective, certains travaux relevant des sciences sociales avaient d'ailleurs vu en L. K. un théoricien de la culture européenne, pratiquant une forme d'anthropologie « at home $e^{10}$ ».

6 Aussi bien l'ouvrage de Marcin Król qui porte principalement sur le style et la pensée de L.K. que les deux volumes d'entretiens plus historiques rassemblés par l'écrivain Zbigniew Mentzel accréditent pour une part cette perspective. Lecteur assidu de L. K., ce dernier avait déjà composé le recueil Éloge de l'inconséquence: textes dispersés des années 1955-1968, publié11 à Londres en 1989. Il avait alors recopié à la main les quatorze kilos de textes qui en composent les trois volumes, avant de leur faire passer clandestinement la frontière. Les deux volumes d'entretiens, réalisés peu de temps avant la mort du philosophe, s'accompagnent d'un effort de documentation 
particulièrement rigoureux et nous livrent des éléments biographiques d'autant plus précieux que L. K. n’a jamais rédigé de mémoires. La conversation lui permet de revisiter son enfance à Radom ainsi que son expérience de la guerre. Son père, résistant "libre penseur ", de gauche, fut assassiné par les Allemands en 1943. L. K. a lui-même habité dans des appartements de Varsovie où se cachaient des Juifs. Il y a notamment croisé Irena Sendler (1910-2009), Juste parmi les nations. Il évoque également les enseignements qui l'ont marqué au cours de ses études de philosophie effectuées dans l'immédiat après-guerre: celui de Tadeusz Kotarbiński dans le domaine de la praxéologie, de Janina Kotarbińska pour la logique, Kazimierz Ajudukiewicz pour la logique mathématique. Plus longuement, le sceptique inconséquent évoque le séminaire d'éthique animé par la grande figure de Maria Ossowska (1886-1974), plus tard interdite d'enseignement, tout comme son mari le sociologue Stanisław Ossowski ${ }^{12}$ (1897-1963) : "d'un côté, elle montrait que la différence entre le bien et le mal, les opinions sur ce qui est bon et mauvais, ce qui est moralement permis et ce qui ne l'est pas, ne sont ni vraies ni fausses, mais d'un autre côté, elle savait parfaitement comment distinguer le bien du mal » (Entretiens, p. 76). On suit l'expérience politique de L. K. à la trace depuis la Libération, tout au long des années d'après-guerre, puis au- delà du départ de la Pologne, à travers ses tribulations d'émigré cosmopolite à Montréal où il rencontre Charles Taylor, à Berkeley, Yale, Paris (François Furet avait tenté de le faire entrer au CNRS), puis Oxford (All Souls) où il est entré grâce au soutien de Isaiah Berlin et Alan Montefiore. Le tout raconté dans un style savoureux. Un exemple? Interrogé sur l'entrée des femmes à All Souls, en 1976, il répond: «j'ai immédiatement inventé une théorie suivant laquelle cette avancée était très dangereuse. Si on laisse entrer les femmes à All Souls, ce sera la fin d'All Souls. Or la fin d'All Souls c'est la fin d'Oxford. La fin d'Oxford c'est évidemment la fin de l'Angleterre. Et la fin de l'Angleterre c'est la fin du monde. Cette théorie fut mise en pièces par mon ami américain Philip Rieff. Selon lui mon raisonnement n'était pas pertinent, car la fin du monde avait déjà au lieu, mais personne ne s'en était encore rendu compte. Finalement, en accord avec les superstitions libérales, j'ai voté pour l'admission des femmes au Collège » (Entretiens, vol. 2, p. 42).

7 Sur l'engagement dans le communisme, on citera cette fois une conversation plus ancienne avec Jerzy Turowicz ("Marxisme, christianisme, totalitarisme», 1988), reprise dans le recueil d'interventions rassemblé par Z. Mentzel, Dans l'entourage des amis : " Comme de nombreux autres amis, ceux dont le parcours est semblable au mien, je régissais assez fortement contre une certaine tradition polonaise, celle que je n'aimais pas : la tradition cléricalo-nationalo-bigote, antisémite, nationaliste [endecka]... (...). Le communisme, tel que je l'imaginais, était la continuation d'une tradition qui m'était plus proche : la tradition rationaliste et cosmopolite, celle de la libre pensée " (p. 98-99). Dans les Entretiens, on apprend que la fin du "révisionnisme» de L. K. ne date pas de la fin des années 60 comme on l'écrit souvent. Dès 1957, celui-ci avait perdu toute illusion de réforme possible du système communiste (vol. 1, p. 172). Au passage, il rappelle qu'il n'utilisait jamais ce mot, hétéro-désigné, pour lui-même: «les termes "révisionnisme", ou "révisionniste" étaient employés depuis la seconde moitié des années 50 par les dirigeants du Parti ou les idéologues officiels des États communistes comme insulte pour stigmatiser les gens qui mettaient en question les dogmes idéologiques, dans le cadre du parti ou dans le cadre du marxisme » (vol. 1, p. 172). Les Entretiens donnent des aperçus émouvants sur les sociabilités des opposants au communisme, des détails parfois rétrospectivement très drôles sur les démêlés avec la 
police qui rappellent immanquablement les récits du voisin Vaclav Havel. Depuis 1963, L. K. est surveillé jour et nuit par les services de sécurité. Après 1966, date de son expulsion du Parti, les agents ne se dissimulent plus. M. Król qui avait été son étudiant se souvient qu'en 1968, L. K. était toujours flanqué de quelques grands gaillards aux airs menaçants. Il donnait alors le cours de philosophie moderne jusqu'aux Lumières, Bronisław Baczko prenant le relais à partir du XvIII ${ }^{e}$ siècle ; tous deux ont été virés du Département de philosophie et de sociologie de l'Université de Varsovie en mars 1968, dans la même fournée que Zygmunt Bauman, Włodzimierz Brus, Stefan Morawski, Marysia Bielińska. Il y eut au cours de ces années mêlant répression du mouvement étudiant/intellectuel et campagne antisémite à grande échelle, de sévères passages à tabac - Stefan Kisielewski, qui avait lancé la célèbre expression «dictature des obscurantistes » [dyktatura ciemniaków] avait été tabassé par des « inconnus »- si bien que des étudiants raccompagnaient parfois L. K. de l'université jusqu'à chez lui.

Il reste qu'au bout d'une vie en partie passée à méditer l'expérience totalitaire, et plus prosaïquement, dans le quotidien des jours, à jouer à cache-cache avec la police communiste, interrogé par Z. Mentzel pour savoir s'il considérait Les principaux courants du marxisme comme son opus magnum, L. K. déclare : « si quelque chose devait constituer mon opus magnum - bien évidemment j'ai conscience du caractère risible de cette expression - ce serait sans doute le plus court de mes ouvrages, à savoir Horror metaphysicus. Je suis également très attaché à mon livre sur Pascal et les jansénistes. [...] J'ai compris au cours de ces recherches - du moins j'ai cru comprendre - différentes questions théologiques d'importance ${ }^{13}$ " (Entretiens, vol.2, p.102). Cette sensibilité intellectuelle n'est pas seulement réactive à l'oppression communiste. Dans l'entretien avec J. Turowicz, cité plus haut, L. K. - que son père n'avait pas fait baptiser, chose relativement rare à l'époque - se souvient que les questions relatives au christianisme et à la religion l'intéressaient depuis sa plus tendre enfance et qu'il poursuivait les lectures des Évangiles et de l'Ancien Testament tout au long de l'occupation (p. 103). Ayant fini par considérer que la chrétienté fait partie intégrante de la culture européenne, il précise : «ce n'est pas la théologie systématique qui m'intéresse. Ce qui m'intéresse c'est l'histoire de la théologie, l'histoire des dogmes, les conflits théologiques et religieux au sein de l'Église, l'histoire de la Réforme, l'histoire des hérésies [...] Je pense que ce que nous trouvons dans la tradition biblique, dans le Nouveau Testament, mais surtout aussi dans les livres de l'Ancien Testament qui m'apparaissent comme de magnifiques monuments inspirés par l'esprit humain, comme le Livre de Job, les Psaumes, certains livres des Prophètes, tout cela nous le comprenons sans la théologie (...), et qu'en sens inverse, la théologie l'obscurcit beaucoup » (p. 104).

9 Il ne s'agit pas de relativiser le courage ni le rôle éminent de L. K. dans l'histoire des luttes politiques qui le mèneront vers l'expulsion du Parti (1966) puis l'exil après 1968, mais de situer le centre de gravité de son œuvre ailleurs. L'analyse du marxisme, du communisme et de la situation polonaise occupe une bonne partie de son travail. Mais comme l'a relevé $\mathrm{M}$. Król, dans un précédent livre, « ses vues politiques n'étaient pas spécialement originales. C'était un libéral modéré avec une grande sensibilité sociale, comme il sied à tout homme décent. En revanche, on lui doit d'excellents textes sur les idées et la culture ${ }^{14} »$.

10 C'est précisément à cette dimension surtout que s'attache le dernier ouvrage de M. Król. Cette excellente biographie intellectuelle est aussi intéressante par la 
personnalité de son auteur dont Esprit avait déjà publié un article ${ }^{15}$ sur L. K. Historien des idées, philosophe politique, M. Król (né en 1944) fut dans les années 80 le rédacteur en chef du mensuel indépendant Res Publica qui a contribué à introduire en Pologne des classiques de théorie politique. Après avoir circulé en samizdat, elle fut la première revue d'opposition à décider de paraître de manière légale en 1987. Dans le paysage de l'opposition polonaise des années 80 , M. Król représente pourrait-on dire une forme de libéralisme conservateur, à condition de préciser que le terme "conservatisme " «se limitait au rapport à la culture ${ }^{16} »$. J. Dewitte souligne bien dans son ouvrage que ce rapport à la culture comme un bien fragile à préserver - et non comme un héritage à liquider - se retrouve chez L. K., et plus généralement chez bon nombre d'intellectuels de l'Autre Europe.

Lorsque M. Król rencontre L. K., celui-ci a 35 ans. Il consacre une partie de son ouvrage au récit de souvenirs et d'anecdotes significatives. À partir des années 70 , son récit cesse d'être chronologique pour entrer davantage dans la substance d'une œuvre progressivement saisie par un questionnement intemporel. Selon M. Król, deux ouvrages importants, presque inconnus en France, constituent à la fois une synthèse des travaux plus anciens et une transition aux textes fondamentaux de L. K. : c'est d'une part le recueil La culture et les fétiches [Kultura i fetysze, Varsovie, 1967], dernier ouvrage de L.K. publié en Pologne avant son exil et d'autre part Présence du mythe [Obecność mitu, Paris, Instytut Literacki, 1972] rédigé en Pologne, mais publié en exil. Dans ce dernier, L.K. se donne pour tâche de «décrire le besoin qui produit des réponses aux questions ultimes, c.-à-d. métaphysiques, c.-à-d. non susceptibles de conversion en questions scientifiques ${ }^{17}$ ». Oublier de telles questions reviendrait à renoncer à l'humanité même de l'homme (M. K. p. 107). Trois enjeux fondamentaux du mythe (religieux ou philosophique) se dessinent ici : le sens, la durée et la continuité. En tant qu'il est source de sens, le mythe est un des moyens d'échapper à ce que L. K. nomme le phénomène d'indifférence du monde. Où se dessine d'ailleurs, selon M. Król, la dimension conservatrice de la pensée de L. K. qui, associant durée des mythes et inertie du conservatisme, estime que l'on ne saurait jamais se passer de l'autorité d'une tradition. Le sacré jouant indubitablement un rôle conservateur, la question que L. K. adresse aux sociétés modernes «est plutôt de savoir comment la société humaine peut survivre sans la présence de forces conservatrices, c'est-à-dire sans la tension perpétuelle entre la structure et le développement. Cette tension est propre à la vie tout court, et si elle devait être une fois écartée, il y a tout lieu de croire que cela signifierait soit la mort par stagnation (au cas où seules les forces de conservation resteraient à l'œuvre) soit la mort par éclatement (au cas où les forces de transformation resteraient seules en jeu, dans le vide structurel ${ }^{18}$ ) ».

Comme le suggère J. Dewitte, toute l'œuvre de L. K. est traversée par la vive conscience d'une tension qui travaille la culture européenne entre deux pôles (doute/certitude) portant chacun deux dangers symétriques (nihilisme/fanatisme). On peut en trouver l'annonce déjà dans "Le prêtre et le bouffon ${ }^{19}$ " (1959) : le premier se fait le gardien de l'Absolu, le second doute de toutes les évidences. Comme d'autres témoins du siècle tels Raymond Aron, L. K. explore incessamment ces deux écueils, tout en suggérant que c'est la déclinaison singulière de l'absolu (du définitif, du normatif, des certitudes enracinées...) et du doute (dénaturalisation des évidences reçues...) qui fait la force de l'aptitude européenne à la mise en question de soi et à la rupture de la clôture ethnocentrique (« Où sont les barbares ? Les illusions de l'universalisme culturel »). 
13 Il lui est arrivé d'écrire que l'une des faces les plus périlleuses de la modernité se situait du côté de l'affaissement des tabous ${ }^{20}$. Pour éviter les mécompréhensions, cette proposition doit être mise en regard avec ses textes critiques à l'égard de toute tendance idéocratique, non seulement celle qui fut à l'œuvre dans le projet totalitaire. D'où par exemple ses prises de position critiques face aux velléités intégristes de certains acteurs du catholicisme polonais au moment de la transition et son positionnement en faveur d'une séparation de l'Église et de l'État. Il déclarait alors non sans malice : «S'il se crée une association de boulangers catholiques, ce n'est pas grave tant qu'ils n'exigent pas que l'on achète le pain uniquement chez eux et qu'ils n'affirment pas que leur pain est meilleur pour des raisons confessionnelles ${ }^{21}$.» On navigue toujours entre Charybde et Scylla. Rien n'est pire que la volonté de démontrer rationnellement sa foi et de l'imposer à tous. Il est mortifère de tenter de faire passer le mythe pour une science. Les religions séculières l'ont amplement documenté. Mais on peut également interpréter le communisme comme une réponse pour échapper au désenchantement du monde, "une convulsion née du besoin désespéré de salut ${ }^{22}$ ». Dans cet ordre d'analyse, l'un des chapitres de Présence du mythe intitulé «le mythe dans la culture des analgésiques ", rappelait déjà lointainement Escape from freedom de Fromm; L.K. y passait en revue différentes formes d'escapisme dans les sociétés contemporaines. Il est donc illusoire de vouloir en découdre définitivement avec les mythes. «La chimère nietzschéenne ou sartrienne, tellement répandue parmi nous, selon laquelle l'homme peut se libérer totalement, se libérer de tout - de toute la tradition et de tout sens préexistant - et qui proclame que tout sens se laisse décréter selon une volonté ou un caprice arbitraires, cette chimère, loin d'ouvrir à l'homme la perspective de l'autoconstitution divine, le suspend dans la nuit ${ }^{23}$ ".

Les chapitres les plus substantiels de l'ouvrage de M. Król portent sur la question du mythe (VI), sur la civilisation (IX), et sur la religion (X). Le livre se clôt sur un bref chapitre XI, intitulé «Philosophie », où l'ensemble de l'œuvre de L. K. est subsumé sous deux préoccupations fondamentales, la question du sens, et celle de la vérité. La question de la vérité et du mensonge totalitaire a une importance cruciale dans les analyses du communisme, on le sait depuis B. Souvarine, A. Ciliga (Au pays du mensonge déconcertant) ou G. Orwell. Le mensonge en politique est banal mais il laisse intacte la distinction entre vérité et fausseté. Au-delà de cette simple logique machiavélienne, le pouvoir totalitaire à travers la destruction de la mémoire et du langage allait beaucoup plus loin en s'efforçant d'« abroger l'idée même de vérité ${ }^{4}$ ». Surtout, c'est l'occasion pour M. Król de relever une fois encore le singulier conservatisme de L. K., qui « traite de la question de la vérité avec un sérieux mortel, non seulement pour des raisons philosophiques, mais surtout parce qu'il saisit parfaitement la simple et éternelle aspiration humaine à savoir ce qui est vrai et ce qui ne l'est pas. D'ailleurs, son rapport à la question du bien et du mal est du même ordre. Même s'il revient fréquemment à l'éventualité de l'illusion, au fait que le diable peut nous égarer (...), il se rend compte néanmoins que les hommes chercheront inévitablement les principes permettant de distinguer le bien du mal. C'est pourquoi, tout en ayant pleinement conscience de l'impossibilité de construire des principes universels, simples à interpréter et applicables à toutes les situations, on ne saurait simplement abandonner la question (...) Le besoin de religion, le besoin de vérité, le besoin de sens se manifestent de fait. Il n'est pas clair si, dans l'esprit de Kolakowski, ils résultent de la nature humaine, mais ils se laissent observer et seuls les aveugles ne les voient pas. Une philosophie qui les négligerait serait donc aveugle » (p. 263). 


\section{NOTES}

1. J. Dewitte a traduit (de l'anglais et de l'allemand) et postfacé, Leszek Kołakowski, L'esprit révolutionnaire, suivi de Marxisme et anti-utopie, Bruxelles, Complexe, 1978, ainsi que Leszek Kołakowski, Le village introuvable, Bruxelles, Complexe, 1986.

2. Główne nurty marksizmu. Powstanie - rozwój - rozkład, Paris, Instytut Literacki, 1976 (t. 1), 1977 (t. 2), 1978 (t. 3) ; trad. du polonais P. S. Falla, Main Currents of Marxism. Its Origins, Growth and Dissolution. Vol. 1. The Golden Age, vol. 2 The Founders, vol. 3. The Breakdown, Oxford, Oxford UP, 1978 ; seuls les deux premiers volumes ont été publiés en français sous le titre Histoire du marxisme, trad. de l'all. O. Masson, Paris, Fayard, 1987 (t. 1 : Les fondateurs, Marx, Engels et leurs prédécesseurs, t. 2 L'âge d'or de Kautsky à Lénine).

3. « Marxist Roots of Stalinism » in Robert C. Tucker (ed.), Stalinism: Essays in Historical Interpretation, New York, Norton, 1977 [trad. fr. in Leszek Kołakowski, Le village introuvable, op. cit.]

4. Sur ce point, voir " Le mythe de l'identité à soi de l'homme. L'unité de la société civile et de la société politique dans la pensée socialiste » (1973) et "Ce qu'on appelle “aliénation" " (1976), repris dans Le village introuvable, op. cit.

5. "Je n'aimais pas le mot "dissident" car il était trompeur. Il désignait quelqu'un qui ne croit plus dans l'idéologie officielle; il devrait donc être utilisé là où des gens y croient. Le communisme dans les pays d'Europe de l'Est après 1968 n'était plus une idée, il n'était que violence ", Entretiens, vol. 2, p. 56.

6. "The Illusion of Demythologization » (1985), repris in Leszek Kołakowski, Modernity on Endless Trial, London, Univ. of Chicago Press, 1990, p. 95-107.

7. «Samozatrucie otwartego społeczeństwa » [L'auto-empoisonnement de la société ouverte] (1979), repris in Leszek Kołakowski, Czy diabeł może być zbawiony i 27 innych kazań [Le diable peut-il être sauvé et 27 autres sermons], London, « Aneks », 1982, p. 206-216.

8. Główne nurty marksizmu. Powstanie - rozwój - rozkład [Paris, Instytut Literacki, t. $1: 1976$, t. 2 :

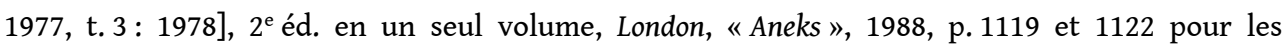
citations; l'ouvrage fut traduit du polonais par P. S. Falla, Main Currents of Marxism. Its Origins, Growth and Dissolution. Vol.1. The Golden Age, vol. 2 The Founders, vol. 3. The Breakdown, Oxford, Oxford UP, 1978 ; seuls les deux premiers volumes ont été publiés en France sous le titre Histoire du marxisme, trad. de l'all. O. Masson, Paris, Fayard, 1987, t. 1 : Les fondateurs, Marx, Engels et leurs prédécesseurs, t. 2 : L'âge d'or de Kautsky à Lénine.

9. Voir aussi Leszek Kołakowski, «Comment une vérité sans Dieu est-elle possible ? Réponse : en aucune manière » (trad. J. Dewitte), Le genre humain, $\mathrm{n}^{\circ}$ 7-8/1983.

10. Mariola Flis, Leszek Kołakowski - teoretyk kutury europejskiej [Leszek Kołakowski - théoricien de la culture européenne], Cracovie Universitas, 1994, p. 7.

11. Leszek Kołakowski, Pochwała niekonsekwencji : pisma rozproszone z lat 1955- 1968, Zbigniew Mentzel (ed.), Londres, Puls, 3 vol., 1989.

12. Sur M. Ossowska en français, Ryszard Kleszcz, « De la pragmatique à la science de la morale », Organon, no $35 / 2006$; signalons le volume de près de 800 pages de correspondance entre les époux, Elżbieta Neyman ed., Intymny portret uczonych. Korespondencja Marii i Stanisława Ossowskich [Portrait intime de savants. La correspondance entre Marie et Stanislaw Ossowski], Varsovie, wyd. Sic !, 2002.

13. L. K. fait ici référence à The Metaphysical Horror, Oxford, Basil Blackwell, 1988 [trad. de l'angl. M. Barat, Horreur métaphysique, Paris, Payot, 1989], ainsi qu'à God Owes Us Nothing: A Brief Remark on Pascal's Religion and on the Spirit of Jansenism, Chicago, The Univ. of Chicago Press, 1995 [trad. de l'angl. A.-M. Lescourret revue par l'auteur, Dieu ne nous doit rien: brève remarque sur la religion de Pascal et l'esprit du jansénisme, Paris, Albin Michel, 1997]. 
14. Marcin Król, Nieco z boku. Autobiografia nie polityczna [Un peu de côté. Autobiographie non politique], Varsovie, Prószyński i S-ka, 2008, p. 54.

15. «Leszek Kołakowski, le philosophe et la religion », Esprit, $n^{\circ}$ 10/1985.

16. Sur ce point, Marcin Król, op. cit., p. 89.

17. Leszek Kołakowski, Obecność mitu, 1972, Varsovie, Prószyński i S-ka, 2005, p. 14.

18. "La revanche du sacré dans la culture profane", 1974, Le besoin religieux (Rencontres internationales de Genève), Neuchâtel, La Baconnière, repris dans La Revue du MAUSS, $\mathrm{n}^{\circ} 22 / 2003$, p. 58.

19. Leszek Kołakowski, « Kapłan i błazen. (Rozważania o teologicznym dziedzictwie współczesnego myś lenia)» [Le prêtre et le bouffon (Réflexions sur l'héritage théologique de la pensée moderne), Twórczość, nº 10/1959.

20. «Cywilizacja na ławie oskarżonych » [La civilisation au banc des accusés], 1985, in Moje słuszne poglądy na wszystko [Mes justes vues sur tout], Cracovie, Znak, 2000, p. 370.

21. Leszek Kołakowski, "Komunizm, Kościót i czarownice » [Le communisme, l'Église et les sorcières], entretien avec Adam Michnik, Gazeta Wyborcza du 21-22 novembre 1992, p. 10-16.

22. «Komunizm jako formacja kulturalna » [Le communisme comme formation culturelle], 1985, repris in Moje słuszne poglądy na wszystko [Mes justes vues sur tout], Cracovie, Znak, 2000, p. 370.

23. Leszek Kołakowski, « La revanche du sacré dans la culture profane », op. cit., p. 60.

24. Leszek Kołakowski « Totalitarianism and the Lie », Commentary, May 1983, p. 535.

\section{AUTEURS}

\section{PAUL ZAWADZKI}

Groupe sociétés, religions, laïcités (GSRL), Université Paris 1, CNRS-EPHE UMR 8582, paul.zawadzki@orange.fr 